\title{
Radial fall of a test particle onto an evaporating black hole
}

\author{
Andreas Aste and Dirk Trautmann \\ Department for Physics and Astronomy, University of Basel, \\ 4056 Basel, Switzerland \\ E-mail: andreas.aste@unibas.ch
}

June 8, 2005

\begin{abstract}
A test particle falling into a classical black hole crosses the event horizon and ends up in the singularity within finite eigentime. In the 'more realistic' case of a 'classical' evaporating black hole, an observer falling onto a black hole observes a sudden evaporation of the hole. This illustrates the fact that the discussion of the classical process commonly found in the literature may become obsolete when the black hole has a finite lifetime. The situation is basically the same for more complex cases, e.g. where a particle collides with two merging black holes. It should be pointed out that the model used in this paper is mainly of academic interest, since the description of the physics near a black hole horizon still presents a difficult problem which is not yet fully understood, but our model provides a valuable possibility for students to enter the interesting field of black hole physics and to perform numerical calculations of their own which are not very involved from the computational point of view.
\end{abstract}

PACS numbers: 04.25.-g; 04.70.-s; 04.70.Dy

The Schwarzschild metric generated by an uncharged non-rotating classical black hole is given by

$$
d s^{2}=\left(1-\frac{r_{s}}{r}\right) c^{2} d t^{2}-\left(1-\frac{r_{s}}{r}\right)^{-1} d r^{2}-r^{2} d \Omega^{2},
$$

where $r_{s}=2 G M_{0} / c^{2}$ is the Schwarzschild radius, $G$ the gravitational constant, $M_{0}$ the mass of the black hole, $c$ the speed of light, and $d \Omega^{2}$ denotes the line element squared on a two-dimensional unit sphere. Due to the Hawking radiation, the black hole evaporates, if we exclude any accretion of energy. For macroscopic black holes, the luminosity is proportional to the inverse mass squared. The corresponding ansatz for the time-dependent black hole mass $M(t)$

$$
\dot{M}(t)=-k M(t)^{-2}
$$

leads directly to

$$
M(t)^{3}=\frac{k}{3}\left(t_{0}-t\right),
$$

where $t_{0}=3 M_{0}^{3} / k$ is the lifetime of the black hole. Accordingly, we have

$$
r_{s}(t)=k^{\prime}\left(t_{0}-t\right)^{1 / 3} \text {. }
$$

It is clear that limited knowledge is available about the actual evaporation process of black holes at present, which is based mainly on the classic works of Unruh and Hawking [1, 2, and there is an ongoing interest in the subject [4, 5, 6. Therefore, our model is purely academic, but we consider it worthwhile to investigate it due to its simplicity and because it exhibits the interesting feature that a particle falling onto a black hole does not vanish from our universe, since it experiences an immediate black hole evaporation. A realistic treatment of the process presented here would involve detailed 
knowledge about the physics in the very close vicinity of the black hole horizon. E.g., Babichev et al. 6] discussed the case of black holes accreting dark energy in the form of a phantom energy, where the energy density $\rho>0$ and the negative pressure $p<0$ fulfill the condition $\rho c^{2}+p<0$. For a black hole surrounded by a constant phantom energy bath, the black hole mass decreases like $(t+\tau)^{-1}$, where $\tau>0$ is a characteristic evolution time. Therefore, we could have chosen decay laws for our discussion given below that differ from eq. (4), but the qualitative results would nevertheless remain the same. It is also clear that equation (3) will fail to describe the evaporation process of the black hole in its final stage.

We also point out that an introduction to the description of evaporating black holes can be found in a known paper by William Hiscock [7]. There, different decreasing functions $M(t)$ in a slightly different framework using the so-called Vaidya metric [8, 9] were considered. The main drawback of using simple approaches like those in [7] is the fact that the impact of the emitted radiation is not taken into account, because the black hole does not evaporate due to the emission of radiation but by artificially adding negative energy to the black hole.

We give here a handwaving argument in order to illustrate the strange physical conditions that might be expected in the vicinity of the black hole horizon. For a distant observer, a massive black hole is a very cold object with Hawking temperature

$$
T_{B H}=\frac{\hbar c^{3}}{4 k_{B} G M}
$$

where $k_{B}$ is the Boltzmann constant. It is well-known that the condition for thermal equilibrium in a static system is $\sqrt{g_{00}(\vec{r})} T(\vec{r})=$ const., where $T(\vec{r})$ is the temperature measured by a local static observer. This condition can be most easily derived when two systems in thermal equilibrium are considered, which are coupled only via their thermal radiation, such that the black body radiation of the two systems has to travel through the gravitational field. For the region near the horizon with $r=r_{s}+\delta$, where $\delta>0$ is small, $g_{00}$ is given approximately by

$$
g_{00}=\left(1-\frac{r_{s}}{r}\right)=\left(1-\frac{r_{s}}{r_{s}+\delta}\right) \simeq \frac{\delta}{r_{s}} .
$$

Furthermore, the spatial distance $d$ to the horizon is

$$
d=\int_{r_{s}}^{r_{s}+\delta}\left(1-\frac{r_{s}}{r}\right)^{-1 / 2} d r \simeq \int_{0}^{\delta} \sqrt{\frac{r_{s}}{\delta}} d \delta=2 \sqrt{r_{s} \delta} .
$$

Therefore, a hypothetical static observer very close to the horizon with $d=l_{P}=\sqrt{\hbar G / c^{3}}$ measures a temperature of the order of the Planck temperature $T_{P}$, since

$$
\frac{k_{B} T_{B H}}{\sqrt{g_{00}\left(\delta=l_{P}^{2} / 4 r_{s}\right)}}=\frac{\hbar c^{3}}{4 G M} \frac{2 r_{s}}{l_{P}}=\frac{\hbar c}{l_{P}}=k_{B} T_{P} .
$$

For an external observer, the test particle gets arbitrarily close to the horizon within finite time, but in contrast to our considerations made above, it is not in a static state, but accelerated, and does therefore not experience the extreme Unruh effect as a static observer. Nevertheless, it will be difficult to make any reasonable predictions about the fate of a particle approaching the horizon, where the physics becomes Planckian.

In the following, we assume that the metric generated by the evaporating black hole is given by expression (11), but with $r_{s}$ replaced by the time-dependent expression given by eq. (44). In view of the very slow evaporation process and the low energy density of the emitted Hawking radiation, this is certainly a satisfactory approximation for our purpose. Near the black hole horizon, the validity of the approximation is dubious at best. 
From now on, we will use geometric units $(\mathrm{G}=\mathrm{c}=1)$ throughout. If we parametrize the (geodesic) worldline of a massive test particle by its eigentime $\tau$, then the equation of motion of the particle falling radially onto the black hole can be derived from the Lagrangian

$$
\mathcal{L}=B(r, t) \dot{t}^{2}-B(r, t)^{-1} \dot{r}^{2}, \quad B(r, t)=\left(1-\frac{k^{\prime}\left(t_{0}-t\right)^{1 / 3}}{r}\right),
$$

where the dot denotes differentiation with respect to the eigentime $\tau$. The Euler-Lagrange equations

$$
\partial_{\tau} \frac{\delta \mathcal{L}}{\delta(\dot{t}, \dot{r})}=\frac{\delta \mathcal{L}}{\delta(r, t)}
$$

then read

$$
\begin{gathered}
\partial_{\tau}(2 B(r, t) \dot{t})=\partial_{t} B \dot{t}^{2}-\partial_{t}\left(B^{-1}\right) \dot{r}^{2}, \\
\partial_{\tau}\left(2 B(r, t)^{-1} \dot{r}\right)=-\partial_{r} B \dot{t}^{2}+\partial_{r}\left(B^{-1}\right) \dot{r}^{2} .
\end{gathered}
$$

Eq. (11) leads to

$$
\begin{gathered}
\ddot{r}=\frac{\partial_{r} B}{2 B} \dot{r}^{2}+\frac{\partial_{t} B}{B} \dot{r} \dot{t}-\frac{B}{2} \partial_{r} B \dot{t}^{2}, \\
\ddot{t}=-\frac{\partial_{r} B}{B} \dot{r} \dot{t}-\frac{\partial_{t} B}{2 B} \dot{t}^{2}-\frac{\partial_{t}\left(B^{-1}\right)}{2 B} \dot{r}^{2} .
\end{gathered}
$$

Additionally, we can take into account that the four-velocity of the test particle is constant

$$
B \dot{t}^{2}-B^{-1} \dot{r}^{2}=1
$$

since we used the eigentime $\tau$ as curve parameter.

We solved eq. (12) in conjunction with eq. (15) numerically. We considered first the analytically solvable case of a particle falling radially on a stable black hole (with mass $M$ ) starting at rest at a distance $R$

$$
\left.\frac{d r}{d \tau}\right|_{r=R}=0, \quad r(\tau=0)=R .
$$

Introducing the parameter $\eta$ defined by

$$
\eta=\arccos \left(\frac{2 r}{R}-1\right)
$$

one obtains

$$
\begin{gathered}
r=\frac{R}{2}(1+\cos \eta), \quad \tau=\sqrt{\frac{R^{3}}{8 M}}(\eta+\sin \eta), \\
t=2 M \sqrt{\frac{R}{2 M}-1}\left[\eta+\frac{R}{4 M}(\eta+\sin \eta)\right]+2 M \ln \left|\frac{\sqrt{\frac{R}{2 M}-1}+\tan \frac{\eta}{2}}{\sqrt{\frac{R}{2 M}-1}-\tan \frac{\eta}{2}}\right| .
\end{gathered}
$$

Such a particle reaches the horizon after an eigentime $\tau_{\mathrm{H}}$

$$
\tau_{\mathrm{H}}=\sqrt{\frac{R^{3}}{4 r_{s}}}\left[\arccos \left(\frac{2 r_{s}}{R}-1\right)+2 \sqrt{\frac{r_{s}}{R}-\frac{r_{s}^{2}}{R^{2}}}\right] .
$$

This value served as a practical independent check for the accuracy of our numerical calculation.

A simple explicit finite difference Euler forward scheme is fully sufficient for the numerical integration of eq. (13), where one can eliminate $\dot{t}$ by using eq. (15). But it is necessary to adapt the eigentime step $\Delta \tau$ during the integration process in order to obtain an accurate solution. As long as 


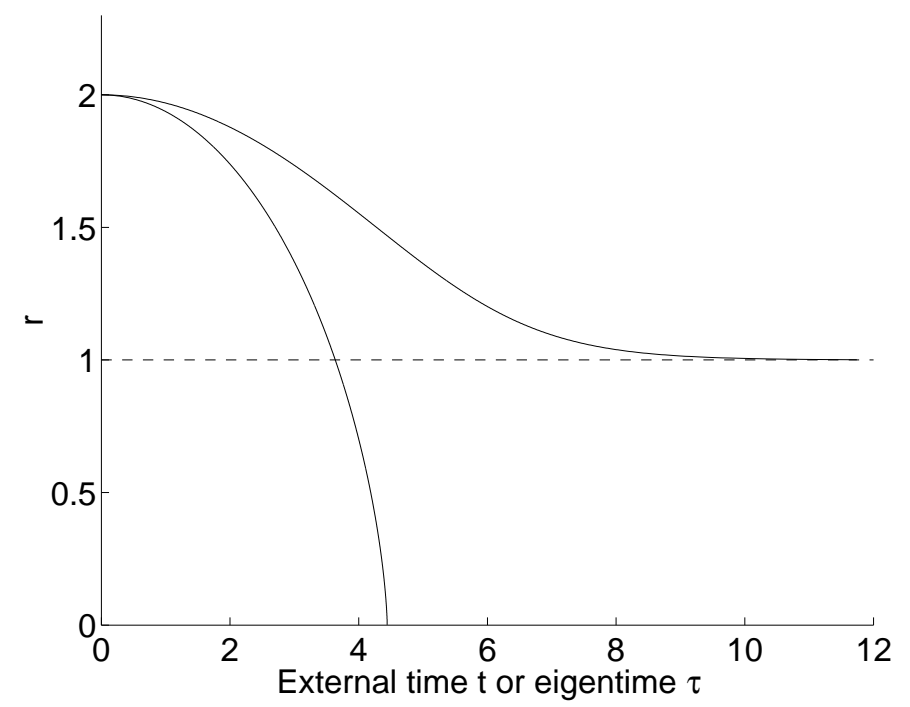

Figure 1: Test particle falling into a stable black hole with Schwarzschild radius $r_{s}=1$. This graph can be found in many standard textbooks.

the particle has a relatively large distance to the horizon, the time step can be held constant, but in the vicinity of the horizon, a update of the time step according to

$$
\Delta \tau_{n+1}=\frac{\dot{r}_{n-1}}{\dot{r}_{n}} \Delta \tau_{n}
$$

for each integration step is a good choice. The effect is a reduction of the time step when the radial coordinate $r$ starts to change faster with respect to the eigentime $\tau$, and the resolution of the trajectory with respect to the external observer time $t$ is maintained.

Fig. 1 shows the trajectory of the particle falling into a stable black hole with $r_{s}=1$ and $R=2$ with respect to the particle's eigentime $\tau$ and the external observer time $t$. In this case, the particle reaches the horizon for $\tau_{\mathrm{H}}=\sqrt{2}(\pi / 2+1) \simeq 3.6357$ and the singularity for $\tau_{\mathrm{S}}=\frac{\pi}{2} \sqrt{R^{3} / r_{s}} \simeq 4.4429$.

Fig. 2 shows the typical situation for an unstable black hole. In order to obtain reasonable plots, we used 'short' black hole lifetimes, but the qualitative picture remains the same independently from the actual lifetime of the black hole which may be very large for massive holes. From the external observer's point of view, the particle impinges on the black hole horizon, where it remains captured until the black hole evaporates. In the final evaporation phase, the particle even looses contact to the horizon due to the fast decrease of the black hole radius.

From the point of view of the test particle, the black hole seems to evaporate as soon as the particle approaches the horizon. The amusing point in our toy model is the fact that from the 'test particle's point of view', the evaporation time of the black hole is larger for black holes with a short lifetime. This is due to the fact that the evaporation process continuously reduces the distance between the horizon to the particle, such that the particle experiences a smaller time contraction near the horizon, when the horizon shrinks faster. For massive black holes, the black hole evaporation time approaches the time that the particle would need to reach the horizon of a stable black hole with $r_{s}=r_{s}(\tau=0)$, given by eq. (19). Fig. 3 shows $\tau\left(t_{0}\right)-\tau_{\mathrm{H}}$, i.e. basically the eigentime of the particle with the same initial conditions as in Fig. 2 at which the evaporation process of the black hole with initial lifetime $t_{0}$ comes to the end.

We conclude with the remark that within our simplified framework one should expect basically the same result for more complex processes, e.g. for a test particle approaching two merging black holes which evaporate subsequently. The test particle cannot cross the classical horizon, and an asymptotic observer will never observe that the particle impinges the horizon within finite time [10]. 

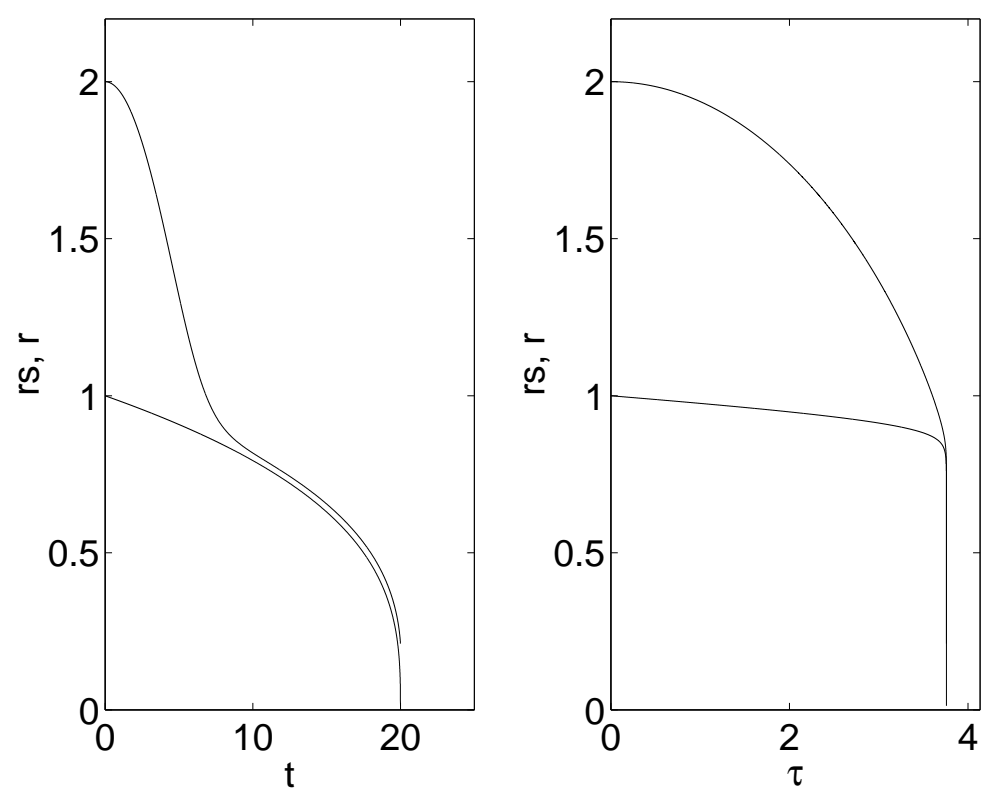

Figure 2: Typical trajectories for a test particle falling onto a black hole with initial Schwarzschild radius $r_{s}(0)=1$ and a lifetime $t_{0}=20$.

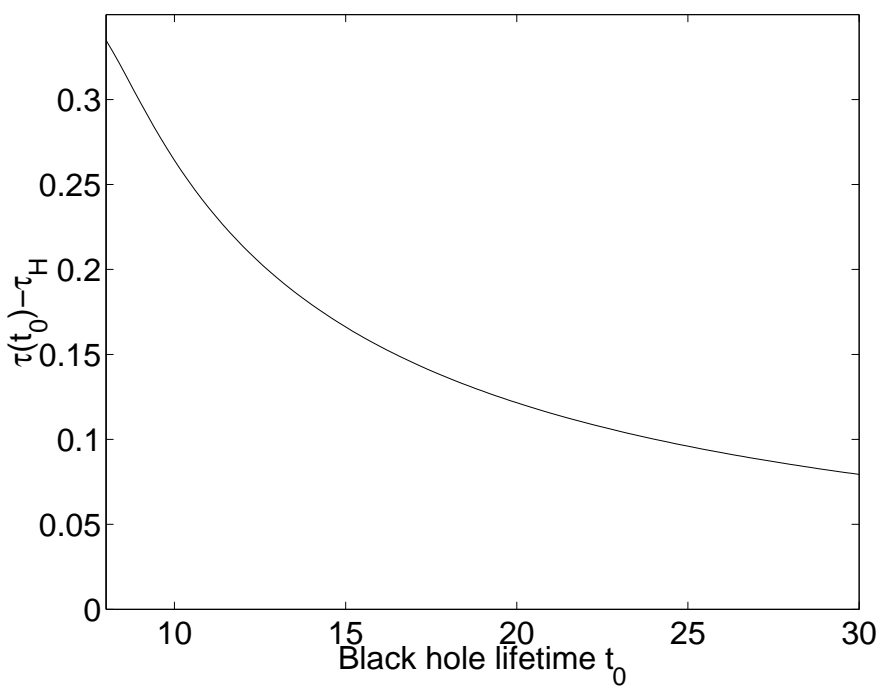

Figure 3: Eigentime $\tau\left(t_{0}\right)$ reduced by the eigentime $\tau_{\mathrm{H}}$ needed by a test particle to reach the horizon of a stable black hole, versus the black hole lifetime $t_{0}$. 


\section{References}

[1] W.G. Unruh, Phys. Rev. D14 (1976) 870.

[2] S.W. Hawking, Commun. Math. Phys. 43 (1975) 199, Nature 248 (1974) 30.

[3] W.G. Unruh, Phys. Rev. D51 (1995) 2827.

[4] T. Jacobson, Phys. Rev. D44 (1991) 1731.

[5] C. R. Stephens, G.'t Hooft, B.F. Whiting, Class. Quant. Grav. 11 (1994) 621.

[6] E. Babichev, V. Dokuchaev, Yu. Eroshenko, Phys. Rev. Lett. 93 (2004) 021102.

[7] W. A. Hiscock, Phys. Rev. D23 (1980) 2813.

[8] P. C. Vaidya, Proc. Indian. Acad. Sci. A33 (1951) 264.

[9] P. Hajicek, W. Israel, Phys. Lett. 80A (1980) 9.

[10] N. Straumann, General Relativity with Applications to Astrophysics, Springer (Berlin) 2004. 